\title{
Tensionless structure of glassy phase
}

\author{
A. Lipowski ${ }^{1)}$ and D. Johnston ${ }^{2)}$ \\ 1) Department of Physics, A. Mickiewicz University, 61-614 Poznań, Poland \\ 2) Department of Mathematics, Heriot-Watt University EH14 4 AS Edinburgh, United Kingdom
}

\begin{abstract}
We study a class of homogeneous finite-dimensional Ising models which were recently shown to exhibit glassy properties. Monte Carlo simulations of a particular three-dimensional model in this class show that the glassy phase obtained under slow cooling is dominated by large scale excitations whose energy $E_{l}$ scales with their size $l$ as $E_{l} \sim l^{\Theta}$ with $\Theta \sim 1.33(5)$. Simulations suggest that in another model of this class, namely the four-spin model, energy is concentrated mainly in linear defects making also in this case domain walls tensionless. Two-dimensinal variants of these models are trivial and energy of excitations scales with the exponent $\Theta=1.05(5)$.
\end{abstract}

Recently the problem of the structure of the glassy phase in spin glasses has attracted considerable attention. The main thrust of research has been to establish whether the low-temperature phase is described by the so-called "droplet model" [1] or by the replica symmetry breaking (RSB) theory of Parisi [2]. An important difference between these theories concerns the energy $E_{l}$ of large-scale excitations which should scale with their size $l$ as $E_{l} \sim l^{\Theta}$ where $\Theta>0$ for the droplet model but with $\Theta=0$ for the RSB theory. The most interesting situation arises in the three-dimensional case, where it seems that a combination of these two approaches is needed to describe the glassy phase correctly [3].

Of course, the problem of the structure of the glassy phase is not restricted to spin glasses. Disordered, out of equilibrium slow dynamics structures appear in superconducting compounds, polymers and granular matter. However, these systems are very complex and modelling them seems to be more difficult than understanding spin glasses. Conventional glasses are also very complex [4]. Nevertheless, recently relatively simple models have been proposed which exhibit an encouraging number of glassy properties [5 90. All these models are spin models which do not contain quenched disorder as in the case of spin glasses and glassiness is dynamically generated. An absence of quenched disorder has important implications. Firstly, their ground state and sometimes even the structure of excitations are known exactly. Let us emphasize that for the three-dimensional spin glasses the problem of finding the ground state is extremely difficult (NP-complete) and is one of the main difficulty in numerical approaches to spin glasses. Secondly, for models without quenched disorder there is no need to average over different realizations of this disorder, which is yet another computational advantage of such models.

The objective of the present paper is to examine the nature of glassy phase in certain nondisordered Ising models. Simple heuristic arguments show that these models might have large scale excitations of energy which scale with their size as $l^{d-2}$, i.e., slower than their surface $\left(\sim l^{d-1}\right)$. The question is whether such states appear in, or maybe even dominate, the glassy phase. To examine this problem we have performed Monte Carlo simulations of the models and our results suggest that the glassy phase is dominated by excitations whose energy increases faster than $l^{d-2}$ but slower than $l^{d-1}$. It is also likely that the exponent $\Theta$ which describes the size dependence of energy of excitations takes some nontrivial values for these models. For the models of conventional glasses considered here the problem of energetics of large scale excitations is computationally much more tractable than for spin glass models. Hopefully, results obtained for these models will provide some insight into other glassy systems too. In addition, our results can be used to verify some earlier claims concerning the nature of the glassy transition in some of these models [10].

The class of models which we examine in this paper is defined by the following Hamiltonian

$$
H=-2 k \sum_{<i, j>} S_{i} S_{j}+\frac{k}{2} \sum_{<<i, j>>} S_{i} S_{j}+\frac{(1-k)}{2} \sum_{[i, j, k, l]} S_{i} S_{j} S_{k} S_{l},
$$

where summations in (1) are over nearest neighbours, next-nearest neighbours, and elementary plaquettes respectively. Model (1) has the interesting property that the energy of cerain excitations of size $l$ is proportional to $l^{d-2}$, whereas typically the energy of an excitation for a standard nearest neighbour Ising model is proportional to its surface $\left(\sim l^{d-1}\right)$.) This property has been used to construct a class of random surface theories based on model (1) [11].

Recently, it was shown that for $d=3$ model (11) has slow dynamics at low temperature [10. When the high temperature sample is quenched to low temperature the excess energy $\delta E=E(t)-E_{e q}$, where $E_{e q}$ is the equilibrium energy, decays with time $t$ much more slowly than $t^{-1 / 2}$, which is a typical decay rate for Ising models with nonconservative dynamics 12]. It turns out that the $k=0$ (pure four-spin interaction) case is of particular interest. This is because in this case the model has also some other properties typical of conventional glasses such as strong metastability [6] and small cooling-rate effects 13. Moreover, certain time dependent correlation functions, such as those describing aging, also behave similarly to real glassy systems [14]. Although a slow decay of $\delta E$ is an indication of slow dynamics, it 
would be desirable to relate this decay with the increase of a characteristic length scale $l$. (As we will see such a relation will give some information about the energetis of excitations of the glassy phase.)

For ordinary Ising models simple arguments, based on the fact that the energy of excitation of the size $l$ scale as its surface $\left(E_{l} \sim l^{d-1}\right)$, can be used to obtain the relation

$$
\delta E \sim \frac{1}{l} .
$$

However, since for model (何) energy of excitations might increase more slowly than their surface area, the relation (2) is no longer obvious. Assuming that in the glassy phase the dominant excitations are indeed these low-energy excitations (with $E_{l} \sim l^{d-2}$ ), the following relation should hold [13]

$$
\delta E \sim \frac{1}{l^{2}}
$$

Let us notice that the assumption that the glassy phase is composed of low-energy excitations implies that at the glassy transition domain walls lose their surface tension. Such an identification might be of more general validity and could be used as a new criterion to locate the glassy transition.

Is it possible to verify which of the relations (2) and (3) are true in our model? First, let us notice that (2) and (3) are two extremal cases corresponding to the largest and the smallest excitation energy per surface area, respectively. It is thus possible that neither of them is true and in the glassy phase an intermediate relation holds. To consider a more general situation let us assume that energy of excitations scale as $l^{\Theta}$. In a lattice of the linear size $L$ the number of excitations of size $l$ scales as $(L / l)^{d}$ and the total excess energy scales as $(L / l)^{d} l^{\Theta}$. Thus, the excess energy per spin $\delta E$ scales as

$$
\delta E \sim l^{\Theta-d} .
$$

To determine $\Theta$ we need a second, independent measurement of the characteristic length $l$. It is already known that $l$ can be also obtained from the fluctuations of the order parameter using the relation [15]

$$
\chi=1 / L^{d}<1 / L^{d}\left(\sum_{i} S_{i}\right)^{2}>=l^{d}
$$

where the magnetization is taken as a corresponding order parameter. Assuming that relation (5) determines the same characteristic length as (4) (up to the order of magnitude), we use the following procedure: We continuously cool the high-temperature sample down to zero temperature. In this process temperature changes linearly with simulation time

$$
T(t)=T_{0}-r t
$$

where $r$ is the cooling rate. Then, for the zero-temperature configuration we calculate $\delta E$ and $\chi$. Previous Monte Carlo simulations suggest that for model (11) and $k=2$ the zero-temperature energy excess $\delta E$ decreases with $r$ as

$$
\delta E \sim r^{x_{1}},
$$

where $x_{1}=0.50(5)$ [13]. Similarly, we expect that $\chi$ also increases as a power of $r$

$$
\chi \sim r^{-x_{2}} .
$$

Inverting (8) and using (5) we obtain $r \sim l^{-d / x_{2}}$ and from (7) we have $\delta E \sim l^{-d x_{1} / x_{2}}$. Finally, comparing the last relation with (雨) we obtain

$$
\Theta=d\left(1-x_{1} / x_{2}\right)
$$

To estimate $\Theta$ we made Monte Carlo simulations of model (1) for $k=2$ and using a sequential Metropolis algorithm [16]. Simulations were made for the system size up to $L=70$ and we have checked that this is sufficient to obtain basically size independent results. For each cooling rate $r$ we made around 100 independent runs which were used to calculate $\delta E$ and $\left.1 / L^{d}<1 / L^{d} \sum S_{i}\right)^{2}>$. The starting temperature was $T=2.8$, which for $k=2$ is above the critical temperature, which in this case is $T_{c} \sim 2.35$ [10].

The results of our simulations are shown in Fig. 1. The relatively good linearity of our data confirm power-law behaviour (7) and (8). From this data we estimate $x_{1}=0.50(5), x_{2}=0.90(5)$ and using (9) we obtain $\Theta=1.33(5)$. 
Such an estimate of $\Theta$ shows that neither (2), which corresponds to $\Theta=2$ nor (3), which corresponds to $\Theta=1$ are correct. Instead, we have an intermediate possibility with a noninteger value of $\Theta$. Let us notice that since $\Theta<2$ the surface tension of domain walls vanishes.

For comparison, in Fig. 1 1 we also present results of our simulations for the two-dimensional (square lattice) version of model (11) with $k=2$ (in this case $T_{c}=0$ ). Simulations were made for system sizes up to $L=1000$. From this data we estimate $x_{1}=0.46(5), x_{2}=0.95(5)$ and thus $\Theta=1.05(5)$. It is likely that in this case $\Theta=1$, which would indicate a trivial nature of domain walls with energy proportional to their perimeter, the typical 2D Ising model behaviour.

Although for $k=2$ model (i1) has slow low-temperature dynamics, it does not display a genuine glassy transition. As we already noted, to model glassy transitions one should really study the case of $k=0$. However, in this case the above method encounters some difficulties since domains of random quench are not only of ferromagnetic type as in the case of $k=2$, but also antiferromagnetic and even of some mixed types (see 10] for some discussion). For $k=0$ equation (5) cannot be used and thus, the exponent $\Theta$ cannot be determined using the above method.

To get some insight into the $k=0$ case we instead looked at the distribution of unsatisfied [17] plaquettes in the glassy phase (i.e., plaquettes contributing energy above the ground state). The random high-temperature sample was slowly cooled down to zero temperature. Then for the final configuration we located unsatisfied plaquettes and their spatial distribution is shown in Fig. 2. For comparison we also present similar calculations for the $k=2$ case. One can see that in both cases energy is concentrated in linear segments. For $k=2$ this is in agreement with our estimation $\Theta<2$ as for $\Theta=2$ energy would be localized on two-dimensional surfaces. Although for $k=0$ we cannot estimate $\Theta$, the linear stuctures in Fig. 2 strongly suggests that in this case also $\Theta<2$ and the glassy phase is composed of tensionless domain walls.

Of course, the glassy phase obtained by the finite-rate cooling contains some regions other than linear segments where energy is concentrated. However, we expect that such spots will diminish for decreasing cooling rate $r$. To confirm our expectations we measured the ratio $p$ of unsatisfied plaquettes which belong to linear segments compared to the total number of unsatisfied plaquettes 18. The results, presented in Fig. 3, show that $p$ indeed increases for decreasing $r$. It is also possible that in the limit $r \rightarrow 0$ the fraction $p \rightarrow 1$. Let us notice that a glassy state obtained in such a limit constitutes an ideal glass [19] and the present results might shed some light on this, so far hypothetical, state of matter. In particular they suggest that in the ideal glass the slow cooling removes energy-rich spots and leaves only low-energy excitations.

In conclusion, we studied the glassy phase of gonihedric model. Our result show that energy of excitations in this phase scales as $l^{\Theta}$ with $\Theta<d-1$. It confirms earlier expectations that domain walls in this model are tensionless. Since the $k=0$ case seems to have a number of properties typical to ordinary glasses, it would be desirable to check whether this result has also some analogy in real systems.

\section{ACKNOWLEDGMENTS}

This work was partially supported by the KBN grant 5 P03B 032 20, the EC IHP network "Discrete Random Geometries: From Solid State Physics to Quantum Gravity" HPRN-CT-1999-000161 and the ESFnetwork "Geometry and Disorder: From Membranes to Quantum Gravity".

[1] D. S. Fisher and D. A. Huse, Phys. Rev. B 38, 386 (1988).

[2] G. Parisi, Phys. Rev. Lett. 43, 1754 (1979); Phys. Rev. Lett. 50, 1946 (1983).

[3] F. Krzakala and O. C. Martin, Phys. Rev. Lett. 85, 3013 (2000). M. Pallasini and A. P. Young, Phys. Rev. Lett. 85, 3017 (2000).

[4] W. Götze, in Liquid, Freezing and Glass Transition, Les Houches Summer School, ed. J. P. Hansen, D. Levesque and J. Zinn-Justin (North-Holland, Amsterdam, 1989). C. A. Angell, Science 267, 1924 (1995). F. H. Stillinger, Science 267, 1935 (1995).

[5] J. D. Shore, M. Holzer and J. P. Sethna, Phys. Rev. B 46, 11376 (1992).

[6] A. Lipowski, J. Phys. A 30, 7365 (1997). A. Lipowski and D. Johnston, J. Phys. A 33,4451 (2000).

[7] M. E. J. Newman and C. Moore, Phys. Rev. E 60, 5068 (1999).

[8] S. Franz, M. Mezard, F. Ricci-Tersenghi, M. Weigt, and R. Zecchina, e-print: cond-mat/0103026

[9] J. Bernasconi, J. Phys. (France) 48, 559 (1987). J. P. Bouchaud and M. Mézard, J. Phys. I (France) 4, 1109 (1994). E. Marinari, G. Parisi and F. Ritort J. Phys. A 27, 7647 (1994). 
[10] A. Lipowski, D. Johnston and D. Espriu, Phys. Rev. E 62, 3404 (2000).

[11] G. K. Savvidy and F. J. Wegner, Nucl. Phys. B 413, 605 (1994). D. Espriu, M. Baig, D. A. Johnston and R. P. K. C. Malmini, J. Phys. A 30, 405 (1997). R. V. Ambartzumian, G. S. Sukiasian, G. K. Savvidy and K. G. Savvidy, Phys.Lett. B 275, 99 (1992).

[12] A. J. Bray, Adv. in Phys. 43, 357 (1994).

[13] A. Lipowski and D. Johnston, Phys. Rev. E 61, 6375 (2000).

[14] M. R. Swift, H. Bokil, R. D. M. Travasso and A. J. Bray, Phys. Rev. B 62, 11494 (2000).

[15] A. Sadiq and K. Binder, J. Stat. Phys. 35, 517 (1984).

[16] See e.g., K. Binder, in Applications of the Monte Carlo Method in Statistical Physics, ed. K. Binder, (Berlin: Springer, 1984).

[17] For $k=0$ a plaquette is called unsatisfied if the product of spin variables on this plaquettes equals 1 . Let us notice that for $k=0$ the coupling in model (11) is positive while that used e.g., in [6, 13] is negative. However, using a simple transformation, both cases can be made equivalent.

[18] A plaquette separates two elementary cubes. This plaquette belongs to the linear segment when each of the adjacent cubes contains only two unsatisfied plaquettes (including the plaquette under consideration).

[19] J. Jäckle, Rep. Prog. Phys. 49, 171 (1986). The idea that in the four-spin model the 'almost ideal' glassy state might exist was discussed in 13.14 .

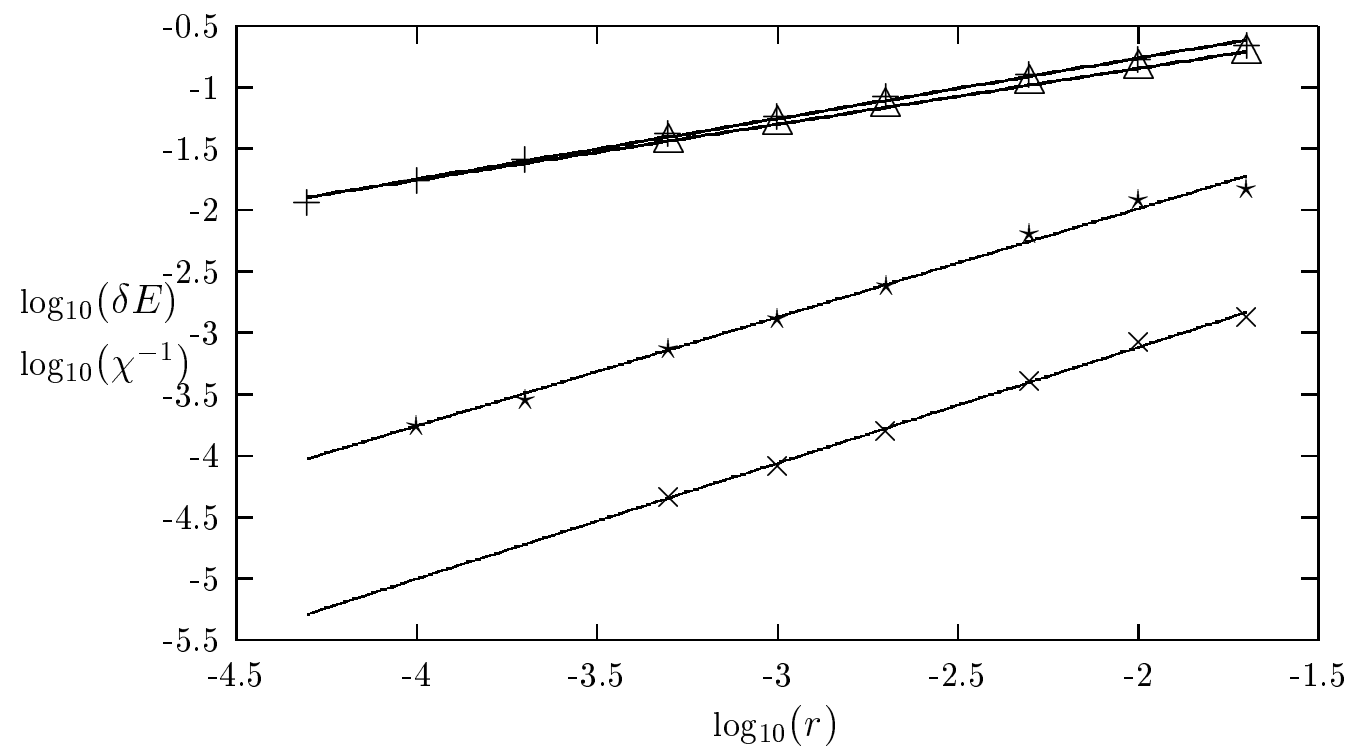

FIG. 1. The excess energy $\delta E(+(d=3)$ and $\triangle(d=2))$, and $\chi^{-1}(\star(d=3)$ and $\times(d=2))$ as a function of the cooling rate $r$. 

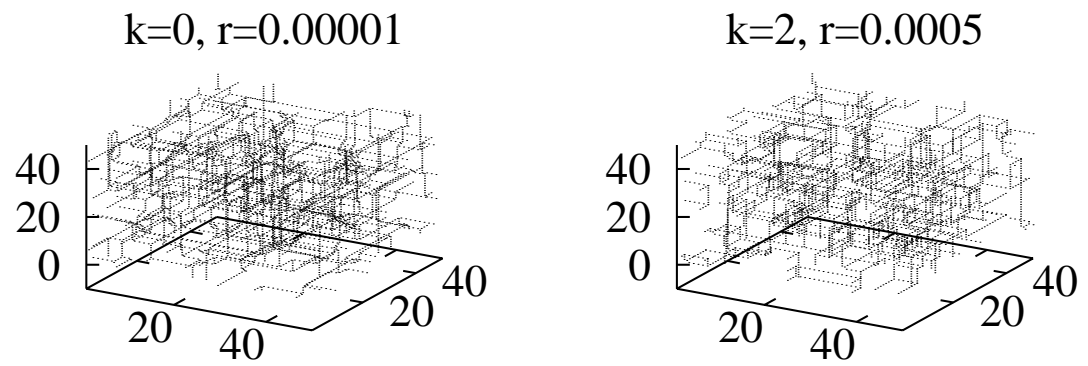

FIG. 2. A distribution of unsatisfied plaquettes in the zero-temperature glassy phase. Simulations were made for the system size $L=100$. (Only a portion of the system is shown.) Let us notice that although the cooling for the $k=2$ case is faster it seems to create larger domains. It was already suggested that for the case $k=0$ the model should order much more slowly than for $k=2[10]$.

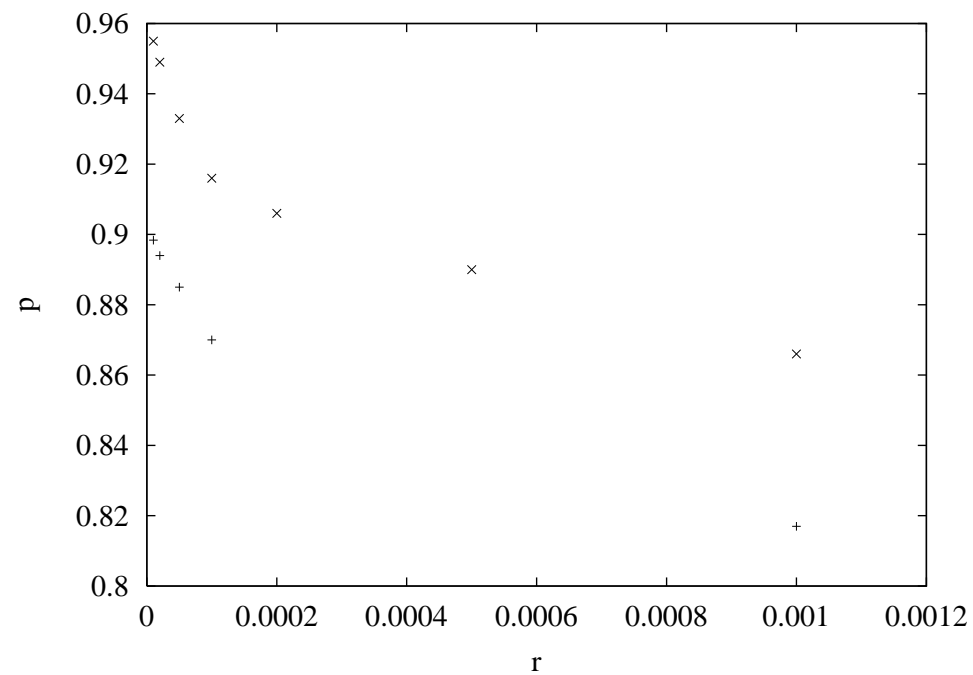

FIG. 3. The fraction $p$ of unsatisfied plaquettes which are located in linear segments as a function of the cooling rate $r$ for $k=0(+)$ and $k=2(\times)$. 